Original research article

\title{
A cardiac troponin I study in a minimally invasive myocardial infarction canine model
}

\author{
Reyaj Mikrani ${ }^{1}$, Cuilan Liang ${ }^{1}$, Muhammad Naveed ${ }^{1}$, Asghar Ali Kamboh ${ }^{2}$, Muhammad Abbas ${ }^{1}$, \\ Birendra Chaurasiya ${ }^{3}$, Li Xue ${ }^{1}$, Zhou Xiaohui 1, 4, 5 * \\ ${ }^{1}$ China Pharmaceutical University, School of Basic Medicine and Clinical Pharmacy, Department of Clinical Pharmacy, Nanjing, China \\ 2 Sindh Agriculture University, Faculty of Animal Husbandry and Vet. Sciences, Department of Veterinary Microbiology, Tandojam, Pakistan \\ ${ }^{3}$ China Pharmaceutical University, Center for Research Development and Evaluation of Pharmaceutical Excipients and Generic Drugs, Nanjing, China \\ ${ }^{4}$ Zhongda Hospital affiliated to Southeast University, Department of Surgery, Nanjing, China \\ ${ }^{5}$ Nanjing Shuiximen Hospital, Department of Surgery, Nanjing, China
}

\section{Abstract}

Cardiac troponin I (cTnI) is an important biomarker of acute myocardial infarction (MI) in animals and human beings. Nevertheless, no immunohistochemical study has been reported about the pattern of myocardial cTnI egression in a minimally invasive model. The present study intended to establish a minimally invasive model of MI and to evaluate the distribution of cTnI. Twelve Mongrel dogs were divided into 2 groups $(n=6)$ : experimental and sham-operated group. Three incisions were made on the left thoracic wall, left anterior descending (LAD) of coronary artery was identified and titanium nips were clamped by video-assisted thoracoscopy surgery (VATS). Series of electrocardiograms (ECG) and biochemical analyses of blood samples - oxidatively modified proteins (OMP), creatine kinase (CK), and cTnI were performed. Furthermore, Masson's trichrome staining was used to observe the histopathology of cardiac myocytes, while immunohistochemistry was done to observe cTnI egression from myocardium. ECG showed elevated ST-segment, whereas OMP, CK and cTnI level increased remarkably and declined to baseline subsequently in the model group throughout study period. Masson's trichrome staining of model group showed a large amount of collagen deposition in the fibrotic area as compared to control group. In immunohistochemical staining, no loss of cTnI staining was observed in non-necrotic myocardium, meanwhile, a great loss was observed in necrotic myocardium. An exception was the myocardium of cardiac apex, where loss of cTnI was visible even in non-necrotic myocardium. All these results revealed that loss of $\mathrm{cTnI}$ occurs not only in the necrotic myocardium but also in so-called non-necrotic myocardium of minimally invasive MI model through VATS.
\end{abstract}

Keywords: Cardiac troponin I; Heart failure; Histopathology; Immunohistochemistry; Myocardial infarction; Video-assisted thoracoscopic surgery

\section{Introduction}

One of the key causes of heart failure (HF) is acute MI with symptoms emerging in those patients who have persistent substantial myocardial injury and ventricular injury (Jhund and McMurray, 2008; Liu and Chen, 2018; Shah et al., 2012). Chest pain in patients at rest without ST-segment elevation on the electrocardiogram, the diagnoses of unstable angina and non-Q-wave MI are generally considered together since they cannot be differentiated clinically or angiographically (Cannon et al., 1995; Fuster et al., 1992; Shukla and Aggarwal, 2017). It has been reported that in the USA alone the overall direct expenses of HF care is more than $\$ 20$ billion per annum (Naveed et al., 2018; Stelzle et al., 2016). The American College of Cardiology/American Heart Association (ACC/AHA) non-ST seg- ment elevation acute coronary syndrome (NSTE-ACS) guidelines in 2014 recommend that Cardiac troponin (cTn) should be measured on first assessment and repeatedly 3-6 h later (Yokoyama et al., 2018). Troponins are proteins involved in the regulation of cardiac and skeletal muscle contraction (Horwich et al., 2003). The cTnI and Cardiac troponin $\mathrm{T}$ (cTnT), are well-established diagnostic and prognostic markers in acute coronary syndromes (Rezvanpour et al., 2014). Cardiac troponin I is highly specific for myocardial tissue and is not detectable in the blood of healthy persons (Adams, 1993a). After an occurrence of myocardial necrosis, it may remain elevated for 7 to 10 days (Adams et al., 1993b). The existence of cardiac troponins in the serum shows myocardial injury or loss of cell membrane integrity (Rainer et al., 2008). The extent of myocardial necrosis is an important determining factor in the risk of death (Kavsak et al., 2007; Rogers et al., 1977).

\footnotetext{
* Author for correspondence: Zhou Xiaohui, China Pharmaceutical University, School of Basic Medicine and Clinical Pharmacy, Department of Clinical Pharmacy, Jiangsu Province, Nanjing 211198, PR China; e-mail: zhxh@cpu.edu.cn http://doi.org/10.32725/jab.2018.001

Submitted: 2018-03-19 • Accepted: 2018-09-18 • Prepublished online: 2018-11-23

J Appl Biomed 17/1: 34-39 • EISSN 1214-0287 • ISSN 1214-021X

(c) 2018 The Authors. Published by University of South Bohemia in České Budějovice, Faculty of Health and Social Sciences.

This is an open access article under the CC BY-NC-ND license.
} 
The introduction of laparoscopic cholecystectomy in the late 1980s marked the beginning of a revolution of minimal access surgery, which had spread to involve almost every surgical subspeciality (Hunter, 1999). Compared with the traditional surgeries, the benefits of minimally invasive procedures include decreased injury and reduced postoperative pain and complications. Minimally invasive trauma could lower the metabolic and immune response (Vittimberga Jr. et al., 1998), since the cytokine network plays a pivotal role in the acute inflammatory and immunologic response to surgical trauma (Vittimberga Jr. et al., 1998; Wan et al., 1997). Several laparoscopic procedures have been shown to be associated with decreased release of cytokines when compared with their open counterparts (Cho et al., 1994; Ellström et al., 1996; Karayiannakis et al., 1997; Sietses et al., 1999; Yuen et al., 1998). So, minimally invasive technique can provide an ideal model for pharmacologists to better understand MI. Since the early 1990s, video-assisted thoracoscopy surgery (VATS) has been proposed to be a less invasive approach than traditional open procedures (Hazelrigg et al., 1993; Kim et al., 2004).

Most authors consider the measurement of $c \operatorname{TnI}$ is a valuable approach for the diagnosis of acute MI and for risk stratification in acute myocardial ischemia (Antman et al., 1996; Apple et al., 1997). However, to date, no immunohistochemical study has been performed to study pattern of myocardial cTnI egression during ischemic injury in a minimally invasive model system. In this work, we have established a minimally invasive MI in canine model through VATS and we have studied the difference in the distribution of $\mathrm{cTnI}$ in the myocardium between the model and sham-operated animals.

\section{Materials and methods}

12 Mongrel dogs, 20-30 months old, weighing 8.7-13.5 kg, were purchased from the Department of Veterinary Clinical Sciences of Tongji Medical College, Huazhong University of Science and Technology China. The animals care and management were performed according to the Principles of Laboratory Animal Care and the Guide for the Care and Use of Laboratory Animals. Animal experimental protocol was approved by the China Pharmaceutical University Institutional Animal Care and Use Committee.

Anti-cardiac Troponin I antibody (ab10231), was bought from Abcam Co., Goat anti-mouse Immunoglobulin G (IgG), $\mathrm{SABC}$ and color-developing reagents were bought from Boster Co., Masson's trichrome staining kit was obtained from Beyotime Institute of Biotechnology, Haimen, China. Titanium nips purchased from Jiangxi Hongda Medical Equipment Group Co. LTD., Nanchang, Jiangxi, China.

\section{Animal model}

We followed our logbook of experiments (2005-2016) to find animal experiments in which well-characterized myocardial injury was formed in a minimally invasive model by VATS (Zhou et al., 2007). Twelve Mongrel dogs were equally divided into two groups: Experimental group and Sham-operated group. Experimental dogs underwent permanent coronary ligation while no ligation was done in sham-operated group. All the dogs were weighed, shaved, scrubbed and disinfected with $75 \%$ ethanol at neck and chest area and anaesthetized by injecting $3 \%$ pentobarbital sodium (30 mg/kg). Anaesthetized dogs were placed in a supine position and endotracheal intubation was performed. Ventilation was acquired by connecting the endotracheal tube to HX-300S ventilator. After achieving steady respiration, three 10 -mm-long incisions on the left thoracic wall were made between the $3 r d$ and 4 th intercostal space and Thoracoport device was placed into the incisions to expose the left ventricle. Thenceforth, LAD of coronary artery and other 1 or 2 diagonal branches were identified and titanium nips were clamped by video-assisted thoracoscopy (McFadden, 2000) to induce MI. The changes of ECG, OMP, CK, and cTnI levels in serum showed that the LADs of coronary arteries in the model group were definitely obstructed by the titanium nips. This ideal minimally invasive animal model of MI could closely resemble the pathophysiological characteristics in patients with remarkable changes of ECG and biochemical parameters etc.

\section{Electrocardiography}

All dogs were anaesthetized with $10 \%$ chloral hydrate and then held in supine position on the worktable and four electrodes of electrocardiogram machine were subcutaneously inserted in the dogs' limbs (Naveed et al., 2017). Briefly, Black electrode was inserted into the right lower limb, Red electrode into the right upper limb, Green electrode into the left lower limb and the Yellow electrode into the left upper limb respectively. A series of ECG was established in all dogs at pre-infarction and post-infarction.

\section{Biochemical parameters}

Series of biochemical analyses of blood sample such as oxidatively modified proteins - OMP (Rutkowska et al., 2005), creatine kinase - CK, and cardiac troponin I - cTnI were performed in the baseline, 1st hour and then in 6th hour, and 1st, 4th, 7th, 14th and 28th day after surgery to assess myocardial injury.

\section{Histopathological analysis}

All the dogs were sacrificed after the completion of study period (28 days). The skin and muscles were clasped and prepared for surgery from the xiphoid process and then their chest and ribs were detached to expose the heart. The aorta were taut with silk suture to avoid bleeding and then their heart was removed. The heart was flushed several times with phosphate buffer saline (PBS) $(1: 100)$ to remove the residual blood and then fixed with $10 \%$ formalin. The heart was then sliced into $5 \mu \mathrm{m}$ coronal sections and dehydrated in graded series of ethanol i.e. 70, 80, 90, 95 and $100 \%$ and fixed in paraffin. Masson's trichrome staining was performed to distinguish the collagen and muscle fibers. We have retrieved the archival paraffin blocks then resliced and prepared one Masson's stained section for each block and examined it for histologic evidence of $\mathrm{MI} /$ necrosis (Kumashiro et al., 1999). Necrosis demarcated as a large amount of collagen deposition in fibrosis area within contraction bands. We have observed the varying degrees of collagen deposition in the necrotic zones. They were comprised of archival formalin-fixed, paraffin-embedded myocardium. The ischemic injury was categorized by following methods that included changes of ECG and OMP, CK, cTnI levels in serum, nitro blue tetrazolium (NBT) staining and identification of Masson's stained sections to show myocardial necrosis.

\section{Immunohistochemical staining}

We have prepared several unstained slides from each paraffin block for immunohistochemical staining (McElroy et al., 1978). All tissues used for immunohistochemical studies resulting from transverse slices of myocardium were fixed in $10 \%$ neutral-buffered formalin for 24-36 h, treated consistently and entrenched in paraffin. We have deparaffinized the 
$5 \mu \mathrm{m}$ tissue sections with xylene two times for $10 \mathrm{~min}$. Slides were rehydrated after 10 min each with 100-70\%-graded alcohol. The activity of endogenous peroxidase was stopped by incubation in 3\% hydrogen peroxidase for $10 \mathrm{~min}$ followed by two times washing with PBS for $5 \mathrm{~min}$. Then sections were incubated for $30 \mathrm{~min}$ with $5 \%$ bovine serum albumin (BSA) (diluted in PBS) at $37^{\circ} \mathrm{C}$ and again incubated at $4{ }^{\circ} \mathrm{C}$ for $12 \mathrm{~h}$ with primary antibodies cTnI (ab10231, $1: 200)$. The primary antibodies were removed by continuous three times washing with PBS for $5 \mathrm{~min}$, then these sections were incubated with goat anti-mouse IgG (1:200) in $1 \%$ NHS for $30 \mathrm{~min}$. After three times washing with PBS for $5 \mathrm{~min}$, sections were incubated with SABC $(1: 1000)$ in PBS for 30 min. After three times washing for $5 \mathrm{~min}$ with PBS, colour-developing reagent was put on the sections, intermittent by the washes of double-distilled water. Counterstaining of slides was done with diluted hematoxylin, rinsed with ammonia and followed with tap water. Graded ethanol series was used for sections dehydration, which were then cleaned in xylene and cover-slipped. Antibody dilutions and incubation time was selected for the optimum staining of tissues.

\section{Statistical analysis}

Statistical analysis was performed using two-way ANOVA to measure statistical differences between data obtained in the control and model groups. All data were presented as mean \pm SEM. The value of $P<0.05$ was considered statistically significant.

\section{Results}

\section{Electrocardiography}

In all dogs of the model group, ST-segment elevation $>0.1 \mathrm{mV}$ was showed on ECG when the last titanium nip was clamped. ST-segment elevation lasted for 28 days. Pathologic $\mathrm{Q}$ waves also showed up in all model animals. No ECG changes were observed in the control group (Fig. 1A).

\section{Biochemical parameters}

To assess myocardial damage, OMP, CK and cTnI levels in serum were measured. In the model group, OMP, CK and CTnI levels were increased remarkably post infarction, reached the peak value at 6-24 h and returned to baseline subsequently. No changes were showed in the control group (Fig. 1B-D).

\section{Histopathological analysis}

To assess the degree of fibrosis, the sections from different parts of hearts were stained with Masson's trichrome staining. A large amount of collagen deposition was found in the fibrosis area of the model group. There was no collagen deposition in non-fibrosis area of the model group and no collagen deposition was observed in the whole area of the sham-operated animals (Fig. 2).

\section{Immunohistochemical analysis}

A significant difference between the model and sham-operated animals was discerned in the levels of serum cTnI. Cardiac troponin I was homogeneously distributed in sham-operated myocardium while it was not seen in the necrotic myocardium. Cardiac troponin I could be found only in the non-necrotic myocardium in the borderline of necrotic and non-necrotic zone of model group but even there, in the non-necrotic myocardium, distribution of $\mathrm{CTnI}$ was not as homogeneous as in the sham-operated myocardium (Fig. 3).
(A)

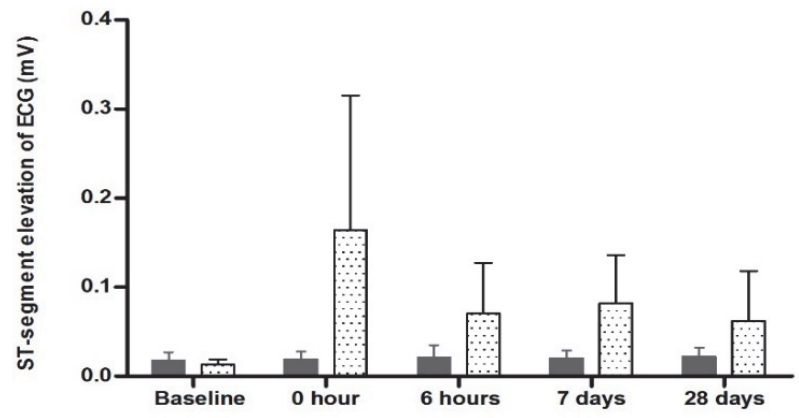

(B)

(C)

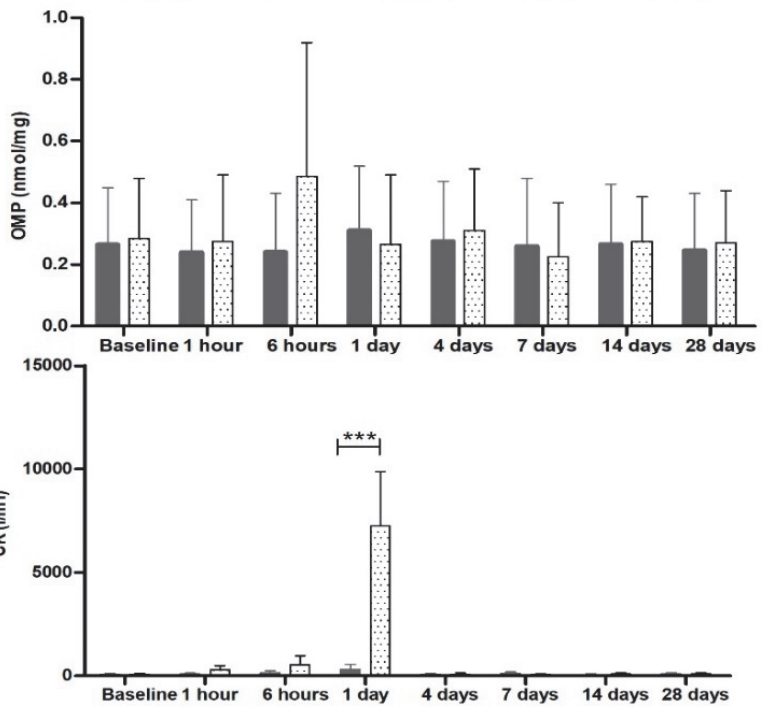

(D)

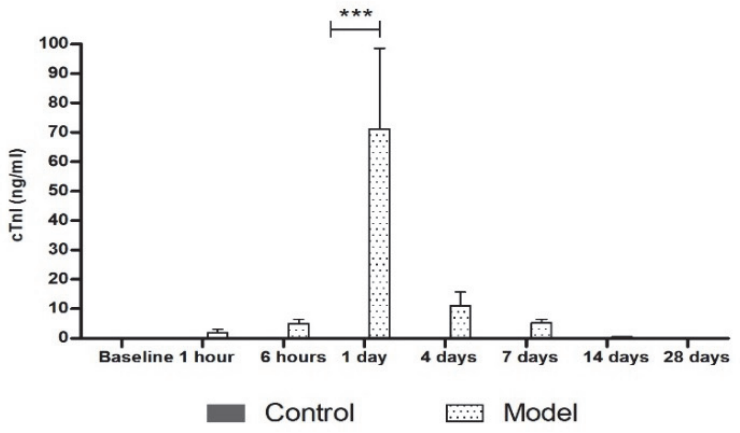

Fig. 1. (A) ST-segment elevation of ECG in pre-operation and post-operation, (B) Oxidatively modified proteins (OMP), (C) creatine kinase (CK) and (D) cardiac troponin I (cTnI) levels in serum, were measured in the control versus model group at different time points $(n=6$, mean \pm SEM, ${ }^{* * *} p<0.001$, two-way ANOVA).

\section{Histopathological and immunohistochemical combination analysis}

Histopathological and immunohistochemical analyses were made together to the myocardium in the cardiac apex of one model dog. The sections of the myocardium in the same position of the same cardiac apex were stained with Masson's trichrome staining and cTnI Immunohistochemical staining (Fig. 4). It was found that loss of cTnI was observed even in non-necrotic zone showed by immunohistochemical staining in the myocardium of cardiac apex.

\section{Discussion}

In the present study, we have found the difference in the distribution of $\mathrm{CTnI}$ in the myocardium between the control and 


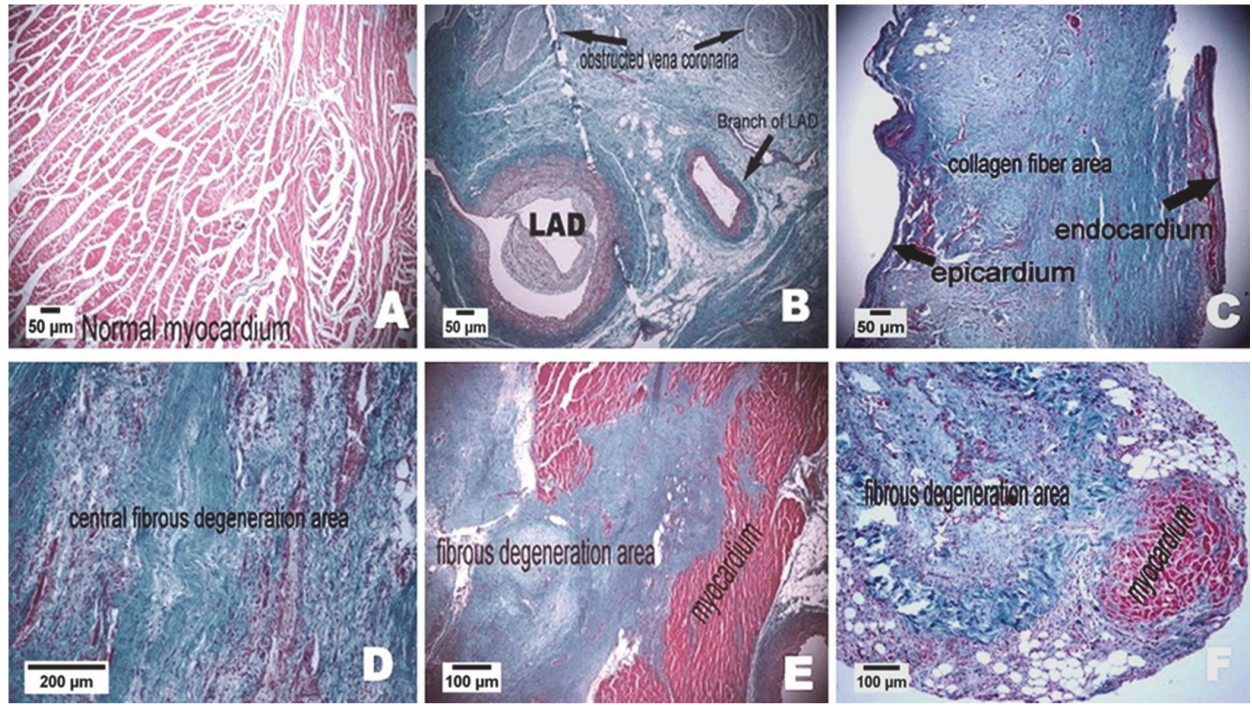

Fig. 2. Masson's trichrome staining of sections of the left ventricular (LV) from dogs sacrificed after 28 days of coronary occlusion: normal myocardium was stained red and collagen fibers were stained green

(A) Sham-operated myocardium. (B) The myocardium tissue below the ligation point. Some small branches of coronary arteries were completely or partially blocked. Most of the myocardium adjacent to coronary artery was replaced by collagen. LAD: left anterior descending of coronary artery.

(C) The central fibrosis area of infarcted myocardium. Full-thickness of myocardium was substituted by collagen. (D) Magnified central fibrous area.

(E) Transitional zones between the normal myocardium and fibrous area. The boundary was map-like. (F) The cardiac apex of the model dogs. Most of the myocardium was fibrous, while there still remained some normal myocardium texture.
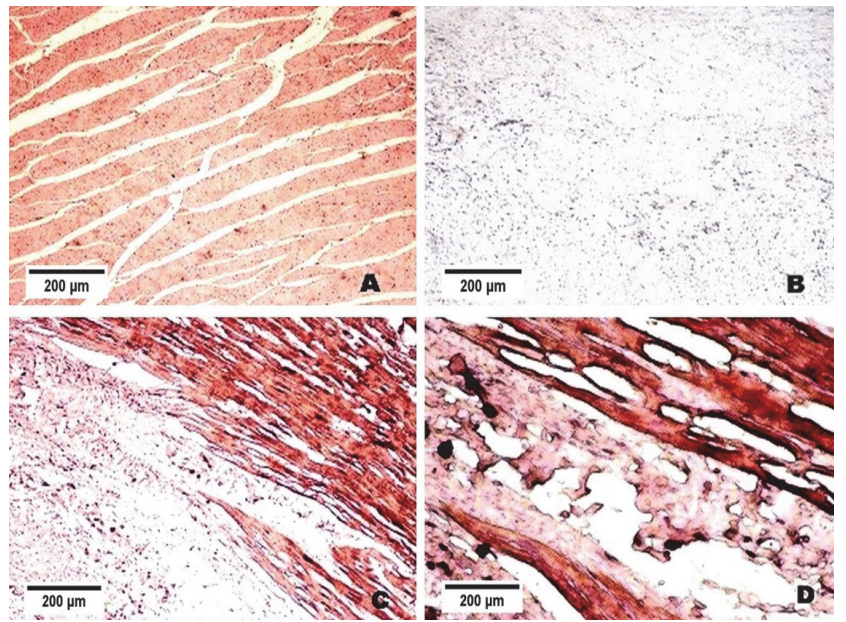

Fig. 3. A cTnI immunohistochemical staining of sections of the LV from dogs sacrificed after 28 days of coronary occlusion: normal myocardium was stained red, and collagen fibers were stained green (A) Sham-operated myocardium: cTnI was in the homogeneous distribution. (B) Necrotic myocardium: cTnI could not be seen in the whole zone. (C) Myocardium in the borderline of necrotic and non-necrotic zone: cTnI could be found only in the non-necrotic myocardium. (D) Magnified picture of $C$ the borderline of necrotic and non-necrotic zone: $c \operatorname{TnI}$ could be found only in the non-necrotic myocardium; but even if in the non-necrotic myocardium, distribution of cTnI was not as homogeneous as in the sham-operated myocardium.

model group through immunohistochemical study. Moreover, we have designed a minimally invasive model of MI by VATS because the invasive injury caused by traditional large opening thoracotomy is so serious, and some of the animal model have difficulties in surviving after surgery, thus this method is not very suitable for carrying out long-time pharmacodynamics study to some extent. The ideal animal model of MI should closely resemble the pathophysiological characteristics in patients with remarkable changes of ECG, biochemical parameters, etc.
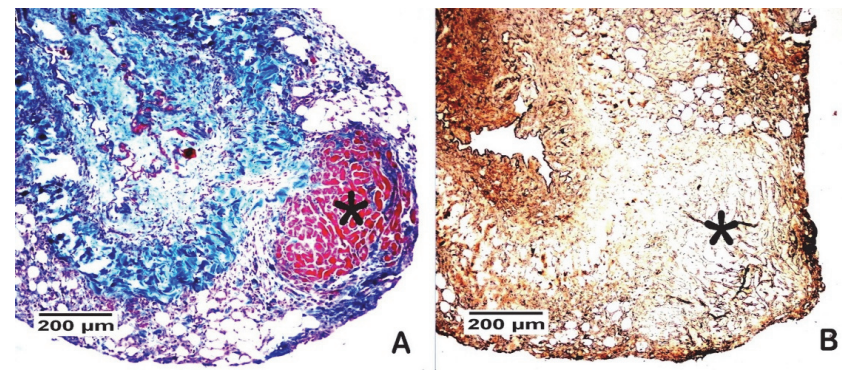

Fig. 4. Masson's trichrome staining and cTnI immunohistochemical staining made to the sections of the myocardium in the same position of the same cardiac apex from one model dog sacrificed after 28 days of coronary occlusion

(A) Masson's trichrome staining of the section of cardiac apex of LV: normal myocardium was stained red, and collagen fibers stained green. Note that there was a patch of "normal" myocardium in a large patch of the necrotic tissue (asterisks). (B) Loss of cTnI was visible in the patch of "normal" myocardium showed by A (asterisks).

We have analyzed a series of biomarkers: changes of ECG and serum levels of OMP, CK and cTnI in control vs. model group. Our result showed that ST-segment elevation of ECG in model group was present up to 28th day of experiment while it was unchanged in sham-operated group. Our results also showed that CK levels were elevated at 24th hour of experiment in model group and returned to baseline quickly, while cTnI was remarkably elevated at 24th hour of experiment and remained elevated till 7 th day. Which is consistent with the results of Feng et al. (1998) who, in the swine model, showed, that the plasma concentrations of cTnl peaked at $18 \mathrm{~h}$, and returned to baseline at 5 th day, while the concentration of creatine kinase-MB isoenzyme peaked at 12 hours after stenosis of the LAD artery and met the baseline at $48 \mathrm{~h}$. Our study is also in accordance with Ricchiuti et al. (1998) in dog model, Leonardi et al. (2008) in ovine model, Benoist et al. (1997) and Jaffe et al. (1996) in humans i.e., cTnI achieved its peak concentration at 1st day of coronary occlusion. These findings clarify the suitability of $\mathrm{CTnI}$ over CK or OMP for detection of 
MI because unlike, CK and OMP, cTnI showed not only sustained elevation but also slow elimination from the circulation after coronary occlusion.

Several studies in different experimental models showed that measurement of cTn in serum is the most sensitive and specific biomarker of myocardial necrosis (Thygesen et al., 2010). Furthermore, various observations suggested that cTn immunohistochemical staining might be useful for identifying myocardial necrosis in human hearts autopsy (Amin et al., 2011; Hansen and Rossen, 1999). Our study, in a minimally invasive canine model of MI, demonstrated that cTnI staining loss could be identified even in the absence of histologic evidence of necrosis such as in cardiac apex, i.e., no loss of cTnI was observed in non-necrotic myocardium, meanwhile, a great loss was observed in necrotic myocardium. An exception was the myocardium of cardiac apex, where loss of cTnI was visible even in non-necrotic myocardium. The explanations for this is, distribution of cTnI flow within the ischemic tissue is not uniform, development of necrosis may occur earlier in some parts of infract than others, as well as flow of cTnI away from necrotic fibers may also be changeable, causing rate of "washout" difference in different fibers (Amin et al., 2011). So, our studies demonstrated that $\mathrm{cTnI}$ immunohistochemical staining may be more sensitive than usual Masson's trichrome staining for the recognition of myocardial necrosis in experimental animals as well as human hearts at autopsy.

Myocardial troponin egress from the myocardium exists in the minimally invasive model of MI, which could be proven by significant difference in the levels of serum cTnI between the model and sham-operated animals. Immunohistochemical staining by antibodies to cTnI can be used to visualize cardiac troponins and record their loss in histologic sections of canine myocardium. To summarize the outcomes with permanent occlusion, antibodies could detect cTnI in normal myocardium and their loss in necrotic myocardium. Fishbein et al. (2003) reported that there was greater loss at the periphery than at the center of the lesions, which we could not observe in this study. We think that its reason is perhaps that the obstructed time of LAD was not a few hours, but 28 days in our study. Thus, cTnI egress got the same degree at the periphery as at the center of the lesions in the last of a very long obstructed time. This phenomenon perhaps resembles the most clinical evidence more likely (Fishbein et al., 2003; Jasra et al., 2012; Jia et al., 2015; Tunca et al., 2008).

\section{Conclusion}

Our results indicated that myocardial troponin, especially cTnI, egress from the myocardium exists in the minimally invasive canine model of myocardial infarction through VATS. Immunohistochemical staining using antibodies to cTnI can be used to visualize pattern of myocardial cTnI egression and its decline in histologic sections of canine myocardium. Loss of cTnI occurs following ischemic injury not only in the necrotic myocardium, but also in so-called non-necrotic myocardium showed by immunohistochemical staining. Furthermore, the present experiment was a minimally invasive pharmacology (MIP) study and perhaps it is a promising animal model for the metabolomics study of MI.

\section{Conflict of interests}

All authors declare no conflict of interests regarding the content of the paper.

\section{Acknowledgements}

This work was supported by the National Nature Science Foundation of China (Grant \#. 30973003 and 30901993) and Administration of TCM (Grant \#. LZ11093) Jiangsu Province, and this work was sponsored by Qing Lan Project and National Found for Fostering Talents of Basic Science (NFFTBS) (Grant \#. J1030830).

\section{References}

Adams JE, Bodor GS, Dàvila-Romàn VG, Delmez JA, Apple FS, Ladenson JH, Jaffe AS (1993a). Cardiac troponin I: A marker with high specificity for cardiac injury. Circulation 88: 101-106.

Adams JE, Abendschein DR, Jaffe AS (1993b). Biochemical markers of myocardial injury. Is $\mathrm{MB}$ creatine kinase the choice for the 1990s? Circulation 88: 750-763.

Amin HAA, El-Hennawy AMY, Nakhla GAA, Tabak SA-H, Hassan HH (2011). Immuno-histochemistry in the detection of early myocardial infarction (a post-mortem study). Egypt J Forensic Sci 1: 5-12. DOI: 10.1016/j.ejfs.2011.04.003.

Antman EM, Tanasijevic MJ, Thompson B, Schactman M, Mccabe CH, Cannon CP, et al. (1996). Cardiac-specific troponin I levels to predict the risk of mortality in patients with acute coronary syndromes. N Engl J Med 335: 1342-1349. DOI: 10.1056/NEJM199610313351802.

Apple FS, Falahati A, Paulsen PR, Miller EA, Sharkey SW (1997). Improved detection of minor ischemic myocardial injury with measurement of serum cardiac troponin I. Clin Chem 43: 2047-2051.

Benoist J-F, Cosson C, Mimoz O, Edouard A (1997). Serum cardiac troponin I, creatine kinase (CK), and CK-MB in early posttraumatic rhabdomyolysis. Clin Chem 43: 416-417.

Cannon CP, Thompson B, McCabe CH, Mueller HS, Kirshenbaum JM, Herson S, et al. (1995). Predictors of non-Q-wave acute myocardial infarction in patients with acute ischemic syndromes: an analysis from the Thrombolysis in Myocardial Ischemia (TIMI) III trials. Am J Cardiol 75: 977-981.

Cho JM, LaPorta AJ, Clark JR, Schofield MJ, Hammond SL, Mallory PL 2nd (1994). Response of serum cytokines in patients undergoing laparoscopic cholecystectomy. Surg Endosc 8: 1380-1384.

Ellström M, Bengtsson A, Tylman M, Haeger M, Olsson J-H, Hahlin M (1996). Evaluation of tissue trauma after laparoscopic and abdominal hysterectomy: measurements of neutrophil activation and release of interleukin-6, cortisol, and C-reactive protein. J Am Coll Surg 182: 423-430.

Feng YJ, Chen C, Fallon JT, Lai T, Chen L, Knibbs DR, et al. (1998). Comparison of cardiac troponin I, creatine kinase-MB, and myoglobin for detection of acute ischemic myocardial injury in a swine model. Am J Clin Pathol 110: 70-77.

Fishbein MC, Wang T, Matijasevic M, Hong L, Apple FS (2003). Myocardial tissue troponins T and I An immunohistochemical study in experimental models of myocardial ischemia Cardiovasc Pathol 12: 65-71. DOI: 10.1016/S1054-8807(02)00188-6.

Fuster V, Badimon L, Badimon JJ, Chesebro JH (1992). The pathogenesis of coronary artery disease and the acute coronary syndromes (1). N Engl J Med 326: 242-250. DOI: 10.1056/ NEJM199201303260506.

Hansen SH, Rossen K (1999). Evaluation of cardiac troponin I immunoreaction in autopsy hearts: a possible marker of early myocardial infarction. Forensic Sci Int 99: 189-196.

Hazelrigg SR, Landreneau RJ, Mack M, Acuff T, Seifert PE, Auer JE, Magee M (1993). Thoracoscopic stapled resection for spontaneous pneumothorax. J Thorac Cardiovasc Surg 105: 389-393.

Horwich TB, Patel J, Maclellan WR, Fonarow GC (2003). Cardiac troponin I is associated with impaired hemodynamics, progressive left ventricular dysfunction, and increased mortality rates in advanced heart failure. Circulation 108: 833-838. DOI: 10.1161/01.CIR.0000084543.79097.34 
Hunter JG (1999). Minimally invasive surgery: the next frontier. World J Surg 23: 422-424.

Jaffe AS, Landt Y, Parvin CA, Abendschein DR, Geltman EM, Ladenson JH (1996). Comparative sensitivity of cardiac troponin I and lactate dehydrogenase isoenzymes for diagnosing acute myocardial infarction. Clin Chem 42: 1770-1776.

Jasra SK, Badian C, Macri I, Ra P (2012). Recognition of early myocardial infarction by immunohistochemical staining with cardiac troponin-I and complement C9. J Forensic Sci 57, 1595: 1600. DOI: 10.1111/j.1556-4029.2012.02172.x.

Jhund PS, McMurray JJ (2008). Heart failure after acute myocardial infarction a lost battle in the war on heart failure? Circulation 118 2019-2021. DOI: 10.1161/CIRCULATIONAHA.108.813493.

Jia JZ, Shen YW, Xue AM, Zhao ZQ (2015). Immunohistochemical analysis of cardiac troponin inhibitor in an experimental model of acute myocardial infarction experimental model and in human tissues. Pathol Res Pr 211: 456-461. DOI: 10.1016/j. prp.2015.01.010.

Karayiannakis AJ, Makri GG, Mantzioka A, Karousos D, Karatzas G (1997). Systemic stress response after laparoscopic or open cholecystectomy: a randomized trial. Br J Surg 84: 467-471.

Kavsak PA, Newman AM, Lustig V, Macrae AR, Palomaki GE, Ko DT, et al. (2007). Long-term health outcomes associated with detectable troponin I concentrations. Clin Chem 53: 220-227. DOI: 10.1373/clinchem.2006.076885.

Kim B-Y, Oh B-S, Jang W, Min Y-I, Park Y-K, Park J-C (2004). Video-assisted thoracoscopic decortication for management of postpneumonic pleural empyema. Am J Surg 188: 321-324. DOI: 10.1016/j.amjsurg.2004.06.009.

Kumashiro H, Kusachi S, Moritani H, Ohnishi H, Nakahama M, Uesugi T, et al. (1999). Establishment of a long-surviving murine model of myocardial infarction: Qualitative and quantitative conventional microscopic findings during pathological evolution. Basic Res Cardiol 94: 78-84.

Leonardi F, Passeri B, Fusari A, De Razza P, Beghi C, Lorusso R, et al. (2008). Cardiac Troponin I (cTnI) concentration in an ovine model of myocardial ischemia. Res Vet Sci 85: 141-144. DOI: 10.1016/j. rvsc.2007.09.010.

Liu C-Y, Chen H-C (2018). A novel algorithm for identifying risk factors for rare events: Predicting transient ischemic attack in young patients with low-risk atrial fibrillation. J Appl Biomed 16: 40-45. DOI: 10.1016/j.jab.2017.10.005.

McElroy CL, Gissen SA, Fishbein MC (1978). Exercise-Induced reduction in myocardial infarct size after coronary artery occlusion in the rat. Circulation 57: 958-962.

McFadden PM (2000). Minimally Invasive Thoracic Surgery. Ochsner J 2: 137-144.

Naveed M, Han L, Khan GJ, Yasmeen S, Mikrani R, Abbas M, et al. (2018). Cardio-supportive devices (VRD \& DCC device) and patches for advanced heart failure: A review, summary of state of the art and future directions. Biomed Pharmacother 102: 41-54. DOI: 10.1016/j.biopha.2018.03.049.

Naveed M, Wenhua L, Gang W, Mohammad IS, Abbas M, Liao X, Yang M, et al. (2017). A novel ventricular restraint device (ASD) repetitively deliver Salvia miltiorrhiza to epicardium have good curative effects in heart failure management. Biomed Pharmacother 95: 701-710. DOI: 10.1016/j.biopha.2017.07.126.

Rainer TH, Chan RW, Graham CA, Yip GW, Yu CM, Wong CK (2008). Comparative performance of heart-type fatty acid-binding protein, myoglobin, troponin $\mathrm{T}$ and electrocardiogram in rapid diagnosis of acute coronary syndrome in chest pain patients with renal impairment presenting to an emergency department. In: International Conference on Emergency Medicine, pp. 481-482.
Rezvanpour A, Shortt C, Clark L, Worster A, Kavsak PA (2014). Analytical factors to consider when assessing a high-sensitivity cardiac troponin I assay compared to a contemporary assay in clinical studies. Clin Chim Acta 429: 6-7. DOI: 10.1016/j. cca.2013.11.012.

Ricchiuti V, Sharkey SW, Murakami MM, Voss EM, Apple FS (1998). Cardiac troponin I and $\mathrm{T}$ alterations in dog hearts with myocardial infarction: correlation with infarct size. Am J Clin Pathol 110: 241-247.

Rogers WJ, McDaniel HG, Smith LR, Mantle JA, Russel RO Jr., Rackley CE (1977). Correlation of angiographic estimates of myocardial infarct size and accumulated release of creatine kinase MB isoenzyme in man. Circulation 56: 199-205.

Rutkowska M, Strzyewski K, Iskra M, Pioruńska-Stolzmann M, Majewski W (2005). Increased protein carbonyl groups in the serum of men with chronic arterial occlusion and the effect of postoperative treatment. Med Sci Monit 11: CR79-83.

Shah RV, Holmes D, Anderson M, Wang TY, Kontos MC, Wiviott SD, Scirica BM (2012). Risk of heart failure complication during hospitalization for acute myocardial infarction in a contemporary population: insights from the national cardiovascular data action registry. Circ Hear Fail 5: 693-702. DOI: 10.1161/ CIRCHEARTFAILURE.112.968180.

Shukla RS, Aggarwal Y (2017). Nonlinear Heart Rate Variability based artificial intelligence in lung cancer prediction. J Appl Biomed 16: 145-155. DOI: 10.1016/j.jab.2017.12.002.

Sietses C, Wiezer MJ, Eijsbouts QAJ, Beelen RHJ, van Leeuwen PAM, von Blomberg BME, et al. (1999). A prospective randomized study of the systemic immune response after laparoscopic and conventional Nissen fundoplication. Surgery 126: 5-9. DOI: $10.1067 / \mathrm{msy} .1999 .98702$.

Stelzle D, Shah A, Denvir M, Anand A, Strachan F, Chapman A, et al. (2016). High-sensitivity Cardiac troponin I and risk of hospitalisation with heart failure in patients with suspected acute coronary syndrome: a cohort study. J Am Coll Cardiol 67: 1327. DOI: $10.1093 /$ ehjqcco/qcx022.

Thygesen K, Mair J, Katus H, Plebani M, Venge P, Collinson P, et al. (2010). Recommendations for the use of cardiac troponin measurement in acute cardiac care. Eur Hear J 31: 2197-2204. DOI: 10.1093/eurheartj/ehq251.

Tunca R, Sozmen M, Erdogan H, Citil M, Uzlu E, Ozen H, Gokce E (2008). Determination of cardiac troponin I in the blood and heart of calves with foot-and-mouth disease. J Vet Diagn Invest 20: 598-605. DOI: 10.1177/104063870802000510.

Vittimberga FJ Jr., Foley DP, Meyers WC, Callery MP (1998). Laparoscopic surgery and the systemic immune response. Ann Surg 227: 326-334.

Wan S, LeClerc J-L, Vincent J-L (1997). Cytokine responses to cardiopulmonary bypass: lessons learned from cardiac transplantation. Ann Thorac Surg 63: 269-276.

Yokoyama H, Higuma T, Endo T, Nishizaki F, Hanada K, Yokota T, Yamada M, Okumura K, Tomita H (2018). "30-minute-delta” of high-sensitivity troponin I improves diagnostic performance in acute myocardial infarction. J Cardiol 71: 144-148. DOI: 10.1016/j.jjcc.2017.08.003.

Yuen PM, Mak TWL, Yim SF, Kee WDN, Lam CWK, Rogers MS, Chang AMZ (1998). Metabolic and inflammatory responses after laparoscopic and abdominal hysterectomy. Am J Obstet Gynecol 179: 1-5.

Zhou X, Li L, Wu L, Wang W, Han L, Yang J, et al. (2007). A minimally invasive model of myocardial infarction made by video-assisted thoracoscopic surgery. Methods Findings Exp Clin Pharmacol 29: 283-290. DOI: 10.1358/mf.2007.29.4.1075359. 